\title{
The Prevalence of Pneumocystis jiroveci in Bronchoalveolar Lavage Specimens of Lung Transplant Recipients Examined by the Nested PCR
}

\author{
Morteza Izadi ${ }^{1}$; Nematollah Jonaidi Jafari ${ }^{1}$; Javid Sadraei ${ }^{2}$; Abbas Mahmoodzadeh Poornaki ${ }^{3}$; \\ Babak Rezavand ${ }^{3, *}$; Hossein Zarrinfar ${ }^{4}$; Jahangir Abdi ${ }^{5}$; Younes Mohammadi ${ }^{6}$ \\ ${ }^{1}$ Health Research Center, Baqiyatallah University of Medical Sciences, Tehran, IR Iran \\ ${ }_{2}^{2}$ Parasitology Department of Medical School, Tarbiat Modares University, Tehran, IR Iran \\ ${ }^{3}$ Department of Parasitology, School of Medicine, Zanjan University of Medical Sciences, Zanjan,IR Iran \\ ${ }_{5}^{4}$ Allergy Research Center, School of Medicine, Mashhad University of Medical Sciences, Mashhad, IR Iran \\ 5 Allergy Research Center, School of Medicine, Mashhad University of Medical Sciences, Mashhad, IR \\ 6 Department of Parasitology, Faculty of Medicine, Ilam University of Medical Sciences, Ilam, IR Iran
Department of Epidemiology \& Bio-statistics, School of Public Health, Tehran University of Medical Sciences, Tehran, IR Iran \\ ${ }^{*}$ Corresponding author: Babak Rezavand, Department of Parasitology, school of Medicine, Zanjan University of medical sciences, Zanjan, IR, Iran. Tel: +98-2414240301-3, E-mail: \\ babak80@gmail.com
}

Received: August 11, 2013; Revised: November 11, 2013; Accepted: February 11, 2014

\begin{abstract}
Background: The use of immune suppressive drugs for organ transplant recipients predisposes them to opportunistic infections, especially by fungal agents. Pneumocystis jiroveci, as an opportunistic pathogen, endangers the patients' life in those with immune system disorders. Early detection of latent Pneumocystis infection in susceptible patients may help choose the optimal treatment for these patients. Objectives: The aim of this study was to identify and determine the colonization of latent $P$. jiroveci infection among lung transplant recipients.

Patients and Methods: This cross-sectional descriptive study was conducted on lung transplant recipients. Bronchoalveolar lavage (BAL) specimens were collected from 32 patients undergoing bronchoscopy. The samples were aseptically homogenized by $10 \mathrm{mM}$ dithiothreitol, and their DNA was extracted. The mtLSUrRNA gene of P.jiroveci was amplified using nested PCR in two stages. Nested PCR was performed using external primers of pAZ-102-E and pAZ102-H followed by using the PCR product of the first stage and internal primers of pAZ-102-E and PAZ102-L2.

Results: The genome of P.jiroveci was revealed by a $346 \mathrm{bp}$ PCR product in the initial amplification and a 120 bp product in the nested PCR The results showed that seven BAL specimens (21.9\%) from lung transplant recipients were positive for P.jiroveci.

Conclusions:In molecular epidemiology studies, nested PCR has higher sensitivity than PCR. Results of this study support the colonization of $P$. jiroveci in patients receiving lung transplantation. Patients who are carriers of $P$. jiroveci are at a higher risk of $P$. jiroveci pneumonia.
\end{abstract}

Keywords:Pneumocystis jiroveci; Lung Transplantation; Nested PCR; Bronchoalveolar Lavage

\section{Background}

Pneumocystis jiroveci is an important determinant of mortality in immunocompromised patients specially AIDS patients (1). Regardless of the source of infection, Pneumocystis can survive and multiply for a long time after entering the body without causing clinical symptoms. After some time, by induction of the immune system of the healthy individual against the organism, multiplication of the infectious agent is controlled, causing infection without symptoms (2). Pneumocystis will evade the immune system surveillance in immunocompromised patients, causing disease symptoms by increasing multiplication (3).

Staining methods and microscopic observation have been considered as the gold standard for diagnosis of Pneumocystis, worldwide (4). One of the disadvantages of microscopic diagnosis is that the patient must be in the final phase of the disease, such that the number of organisms in respiratory samples must reach a threshold to be visible and diagnosed (5). Bronchoalveolar lavage (BAL) specimens have the highest sensitivity among non-invasive samples in detection of $P$. jiroveci pneumonia (PJP) (6).P.jiroveci pneumonia can be controlled and treated in its early stages. Therefore, quick and timely detection of latent Pneumocystis in susceptible patients is very important for clinicians (7). The use of molecular methods can play an important role in identification and biological control of pneumocystosis (8). The first lung transplant in Iran was performed in 2001, after which many patients have received a lung transplant in this country (9). Lung transplant recipients are susceptible to develop a range of opportunistic infectious diseases. Screening for opportunistic infectious agents in transplant recipients can be directly correlated with patient's health $(10,11)$.

\section{Objectives}

Given the importance of molecular epidemiology studies, this study aimed to determine the colonization of

Copyright (C) 2014,Ahvaz Jundishapur University of Medical Sciences; Published by Kowsar. This is an open-access article distributed under the terms of the Creative Commons Attribution-NonCommercial 4.0 International License (http://creativecommons.org/licenses/by-nc/4.0/) which permits copy and redistribute the material just in noncommercial usages, provided the original work is properly cited. 
Pneumocystis among lung transplant patients undergoing bronchoscopy.

\section{Patients and Methods}

\subsection{Patients}

This cross sectional study was conducted on 32 BAL specimens of lung transplant recipients undergoing bronchoscopy under a physician's orders due to respiratory problems. Samples were collected from several specialized hospitals in Tehran from September 2010 to October 2012, after obtaining an informed consent from both the patients and physicians. All the patients were negative for tuberculosis and HIV.

\subsection{DNA Preparation and Extraction}

Bronchoalveolar lavage specimens were collected in sterile conditions and homogenized by $10 \mathrm{mM}$ dithiotritol (12). Genomic DNA was extracted using the MTB extraction kit manufactured by Roche.

\subsection{Primers}

Genome amplification was done using initial primers pAZ102-E: 5'-GATGGCTGTTTCCAAGCCCA-3' and pAZ102-H: 5'-GTGTACGTTGCAAAGTACTC-3' (13). Next, the initial PCR product was amplified by internal primers pAZ102-E and pAZ102-L2: 5'-ATAAGGTAGATAGTCGAAAG-3' (14).

\subsection{PCR}

Polymerase chain reaction was performed in a final volume of $25 \mu \mathrm{L}$ by adding $1 \mu \mathrm{L}$ of $10 \mathrm{pM}$ primers, $0.8 \mu \mathrm{L}$ magnesium chloride, $0.8 \mu \mathrm{L}$ dNTP, $0.8 \mu \mathrm{L}$ Taq DNA, $2.5 \mu \mathrm{L}$ of PCR buffer and $2.5 \mu \mathrm{L}$ DNA template. The thermal protocol of the PCR was as follows: four minutes of initial denaturation, 30 seconds for denaturation of DNA strands, primer binding for initial PCR for 30 seconds at $59.5^{\circ} \mathrm{C}$ and $56^{\circ} \mathrm{C}$ for the secondary amplification, 30 seconds at $72^{\circ} \mathrm{C}$ for extension in 35 cycles, four minutes at $72^{\circ} \mathrm{C}$ for final extension. The amplified PCR product was run on $1.5 \%$ electrophoresis on gel, and was assessed in the transilluminator device after staining with ethidium bromide.

\subsection{Negative and Positive Controls}

In the present study, the positive control was a sample of $P$. jiroveci isolated and sequenced from HIV positive patients in Iran with code number JF733748, listed on the World Gene Bank (15). Deionized distilled water was used instead of a template as the negative control.

\subsection{Statistical Test}

Kappa test was used to measure the agreement between PCR and nested PCR. Fisher's exact test was used to assess the significance between gender, duration of lung transplant and presence of $P$. jiroveci DNA. Independent t-test was used to assess the significance between age and presence of P. jiroveci DNA. All differences were deemed significant at the level of $\mathrm{P}<0.05$. Confidence interval of 95\% was considered for all tests. All statistical analysis was performed using the Statistical Package for the Social Sciences (SPSS) version 18.0 software (Chicago, Inc., USA).

\section{Results}

Of the 32 patients under study, there were 24 (75\%) males and eight (25\%) were females. The average age of patients was $32 \pm 8.5$ years. Pneumocystis specific DNA amplification was performed, using Paz-102E and Paz-102H primers on 32 BAL specimens of lung transplant patients, and had positive results for four patients (12.5\%). The next step was specific genome amplification of $P$. jiroveci using Paz-102E and Paz-L2 primers from the initial PCR product. Seven samples (21.9\%) were positive for $P$. jiroveci in BAL specimens (Table 1). Positive samples in $1.5 \%$ agarose gel electrophoresis produced a $347 \mathrm{bp}$ band in the initial amplification and 120 bp bands in the second amplification, as indicated by Figure 1.

PCR and nested PCR in all 32 patients enrolled in this study indicated four positive cases of $P$. jiroveci DNA and 25 negative cases. Based on the kappa test, there was $68 \%$ agreement between PCR and nested PCR, which is a fairly good degree of agreement. The mean age of negative and positive patients for Pneumocystis DNA using nested PCR was 31.56 and 33.85 years, respectively. No significant correlation was found between positive nested PCR and the patients' age, using the independent t-test $(\mathrm{P}=0.54)$. Of the 24 male patients under study, there were five patients (20.8\%) positive for the P.jiroveci genome, and 19 patients (79.2\%) were negative. Among the eight female patients in the study, two patients (25\%) were positive for the $P$. jiroveci genome and six patients (75\%) were negative. The fisher exact test indicated that there was no significant relationship between the presence of $P$. jiroveci genome and patient's gender $(\mathrm{P}=0.99)$.

Among the 18 patients who had received a lung transplant within the last six months, four patients $(22.2 \%)$ were positive for the $P$. jiroveci genome. From 14 lung transplant patients whose transplant duration was more than six months, three patients (21.4\%) were positive for the P.jiroveci genome. Using the fisher's exact test, there was no significant relationship between duration of lung transplant and presence of $P$. jiroveci genome $(\mathrm{P}=0.99)$.

\section{Discussion}

Treatment protocol for patients with various malignancies and organ transplant recipients may involve immunosuppressive agents such as corticosteroids, cyclosporine or cyclophosphamide. All these drugs act on both arms of the immune system as immunosuppressive agents $(16,17)$. Control of Pneumocystis infection in people with healthy immune systems is mainly dependent on $\mathrm{T}$ cell activity, but the role of humoral immunity has been 
Izadi $M$ et al.

\begin{tabular}{|c|c|c|c|c|c|c|}
\hline \multirow[b]{2}{*}{ Transplantation Time } & \multicolumn{3}{|c|}{ Single Round PCR No. (\%) } & \multicolumn{3}{|c|}{ Nested PCR No. (\%) } \\
\hline & Positive & Negative & Total & Positive & Negative & Total \\
\hline Less than 6 months & $1(25 \%)$ & $17(60.7 \%)$ & $18(56.2 \%)$ & $4(57.1 \%)$ & $14(56.0 \%)$ & $18(56.2 \%)$ \\
\hline More than 6 months & $3(75 \%)$ & $11(39.3 \%)$ & $14(43.8 \%)$ & $3(42.9 \%)$ & $11(44.0 \%)$ & $14(43.8 \%)$ \\
\hline Total & $4(100 \%)$ & $28(100 \%)$ & $32(100 \%)$ & $7(100 \%)$ & $25(100 \%)$ & $32(100 \%)$ \\
\hline
\end{tabular}

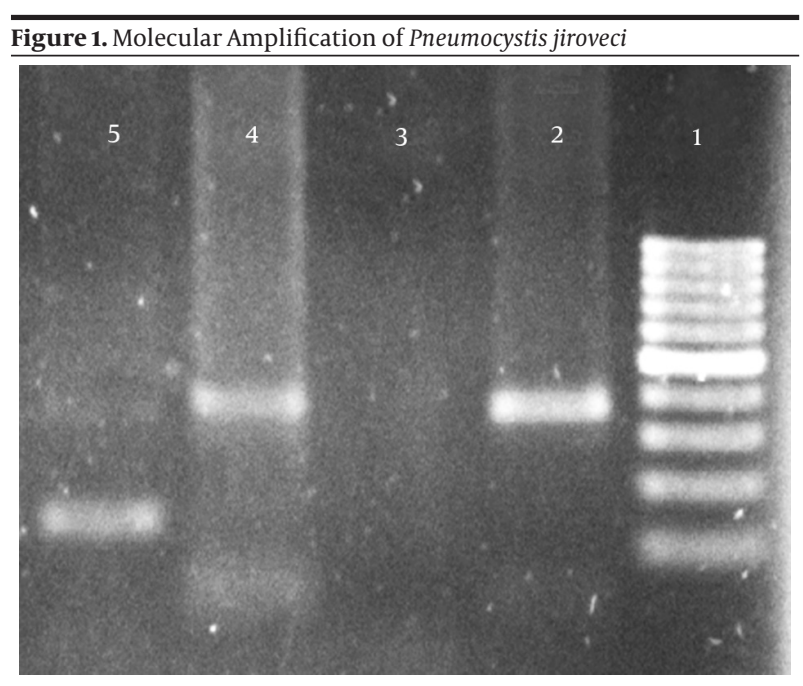

Lane 1: 100 bp marker, Lane 2: positive control, Lane 3: negative control, Lane 4: positive control with general primers for the whole Pneumocystis family, Lane 5: positive samples amplified from initial PCR product with Pneumocystis specific primers.

indicated by experimental and clinical data $(2,18)$. Great deal of studies show that the presence of Pneumocystis can be dangerous in susceptible patients, so that presence of Pneumocystis specific amplicons from oral cavity swabs of adult rats without immunosuppression can predict incidence of $P$. carinii pneumonia (PCP) after immunosuppression, due to corticosteroid administration (19). The first epidemic of Pneumocystis pneumonia was reported between 1961 and 1963 in two orphanages in Shiraz by Post et al.; from the 168 studied children, 40 died and the presence of Pneumocystis was demonstrated in six patients (12.5\%) by autopsy (20). Appropriate prophylaxis against $P$. jiroveci infection is valuable in susceptible patients. In 1971, Post et al. used sulfadoxine pyrimethamine as a prophylaxis in children at risk of Pneumocystis pneumonia in Shiraz orphanages, and the incidence of disease was decreased to $0 \%$ from $25 \%$ in the control group (21).

In AIDS patients, the risk of PJP is rapidly increased when the number of CD4 + lymphocytes decreases to $200 \times 10^{6}$ per $\mathrm{mL}$. The incidence of PJP is five times higher in those with a CD4 + lymphocyte count of less than $200 \times 10^{6}$ compared to patients with CD $4+$ counts higher than this value $(22,23)$. P. jiroveci mortality in HIV positive patients in Iran has been reported to be up to $26.6 \%$ (15). Treatment protocol of $P$. jiroveci among transplant patients is co-trimoxazole consumption three to six months posttransplant (24). P. jiroveci even colonizes healthy individ- uals free from any respiratory problems. The incidence of $P$. jiroveci in respiratory samples of healthy subjects has been reported to be up to $20 \%$. Latent Pneumocystis colonization is directly related to the immune system status of the individual (25). P. jiroveci colonization is higher in HIV negative patients with respiratory problems consuming glucocorticoids (26). There are many reports of PJP among patients receiving kidney, heart, bone marrow and lung transplants $(27,28,29)$. Molecular identification of $P$. jiroveci has been done in respiratory specimens of patients with malignancies and respiratory problems. Molecular screening of $P$. jiroveci in pulmonary lavage samples of non-HIV infected patients in Iran indicates that $P$. jiroveci colonization is higher in transplant recipients and cancer patients compared to other patients (29). PCR based diagnosis of Pneumocystis in respiratory specimens is a valuable tool for screening of this organism. Single step PCR cannot precisely detect the infectious agent in some cases, especially in low template DNA conditions. Therefore, in this study the more sensitive and reliable nested PCR was used due to its ability to amplify very small quantities of DNA (31). In this study, four samples in PCR and four samples in the nested PCR were positive for $P$. jiroveci. A great deal of studies support the presence of latent $P$. jiroveci in respiratory specimens of patients with malignancies and respiratory problems. There are evidences of $P$. jiroveci colonization in respiratory specimens of healthcare staff in contact with PJP (32). Several studies indicate transmission of $P$. jiroveci by air droplets in humans. P. jiroveci has been isolated from ambient air in rooms of patients with PJP using the PCR method (33). Considering the duration of hospitalization of lung transplant recipients, isolating the air in the rooms of these patients seems to be necessary. According to the results of this study, a remarkable presence of P.jiroveci is evident in respiratory samples of lung transplant patients. Therefore, prophylaxis against Pneumocystis is recommended according to available standards.

\section{Acknowledgements}

The authors acknowledge Dr. Mirhendi for providing the samples.

\section{Authors' Contributions}

Study design: Morteza Izadi, Babak Rezavand and Nematollah Jonaidi Jafari. Statistical analysis: Younes Mohammadi and Jahangir Abdi. Sample collection: Babak Rezavand and Hossein Zarrinfar. PCR procedures: Babak Rezavand. Manuscript wiring and preparation: Babak Rezavand, Javid Sadraei, Morteza Izadi and Abbas Mah- 
moodzadeh Poornaki. PCR and slide analysis: Babak Rezavand, Abbas Mahmoodzadeh Poornaki and Morteza Izadi.

\section{Funding/Support}

This study was supported by grant no. 378 from the Medicine Faculty of Baqiyatallah University of Medical Sciences.

\section{References}

1. Patel N, Koziel H. Pneumocystis jiroveci pneumonia in adult patients with AIDS: treatment strategies and emerging challenges to antimicrobial therapy. Treat Respir Med.2004;3(6):381-97.

2. Kelly MN, Shellito JE. Current understanding of Pneumocystis immunology. Future Microbiol. 2010;5(1):43-65.

3. Azoulay E, Bergeron A, Chevret S, Bele N, Schlemmer B, Menotti J. Polymerase chain reaction for diagnosing pneumocystis pneumonia in non-HIV immunocompromised patients with pulmonary infiltrates. Chest. 2009;135(3):655-61.

4. Cregan P, Yamamoto A, Lum A, VanDerHeide T, MacDonald M, Pulliam L. Comparison of four methods for rapid detection of Pneumocystis carinii in respiratory specimens. J Clin Microbiol. 1990;28(11):2432-6.

5. Carmona EM, Limper AH. Update on the diagnosis and treatment of Pneumocystis pneumonia. Ther Adv Respir Dis. 2011;5(1):41-59.

6. Pinlaor S, Mootsikapun P, Pinlaor P, Phunmanee A, Pipitgool V Sithithaworn P, et al. PCR diagnosis of Pneumocystis carinii on sputum and bronchoalveolar lavage samples in immuno-compromised patients. Parasitol Res. 2004;94(3):213-8.

7. Thomas CF, Jr, Limper AH. Pneumocystis pneumonia. $N$ Engl J Med. 2004;350(24):2487-98.

8. Beard CB, Navin TR. Molecular epidemiology of Pneumocystis carinii pneumonia. Emerg Infect Dis. 1996;2(2):147-50.

9. Broumand B. Transplantation activities in Iran. Exp Clin Trans plant. 2005;3(1):333-7.

10. Alexander BD, Tapson VF. Infectious complications of lung transplantation. Transpl Infect Dis. 2001;3(3):128-37.

11. Grossi P, Farina C, Fiocchi R, Dalla Gasperina D. Prevalence and outcome of invasive fungal infections in 1,963 thoracic organ transplant recipients: a multicenter retrospective study. Italian Study Group of Fungal Infections in Thoracic Organ Transplant Recipients. Transplantation. 2000;70(1):112-6.

12. Govender S, Du Plessis SJ, Ocana GS, Chalkley LJ. Prevalence of Pneumocystis jirovecii and Mycoplasma pneumoniae in patients presenting with pneumonia at hospitals in Port Elizabeth: original research. South Afr J Epidemiol Infect. 2008;23(2):21-4.

13. Wakefield AE, Pixley FJ, Banerji S, Sinclair K, Miller RF, Moxon ER, et al. Detection of Pneumocystis carinii with DNA amplification. Lancet.1990;336(8713):451-3.

14. Wakefield AE, Pixley F, Banerji S, Sinclair K, Miller RF, Moxon ER, et al. Amplification of mitochondrial ribosomal RNA sequences from Pneumocystis carinii DNA of rat and human origin. Mol Biochem Parasitol.1990;43(1):69-76.

15. Sheikholeslami FM, Sadraei J, Farnia P, Forozandeh M, Kochak HE. Rate of Pneumocystis pneumonia in Iranian HIV+ Patients with Pulmonary Infiltrates. Jundishapur J Microbiol. 2013;6(3):295-300.

16. Gleichmann E, Kimber I, Purchase IF. Immunotoxicology: suppressive and stimulatory effects of drugs and environmental chemicals on the immune system. A discussion. Arch Toxicol. 1989;63(4):257-73.

17. Ehrke MJ, Mihich E, Berd D, Mastrangelo MJ. Effects of anticancer drugs on the immune system in humans. Semin Oncol. 1989;16(3):230-53.

18. Vahid B, Bibbo M, Marik PE. Role of CD8 lymphocytes and neutrophilic alveolitis in Pneumocystis jiroveci pneumonia. Scand Infect Dis. 2007;39(6-7):612-4.

19. Icenhour CR, Rebholz SL, Collins MS, Cushion MT. Early acquisition of Pneumocystis carinii in neonatal rats as evidenced by PCR and oral swabs. Eukaryot Cell. 2002;1(3):414-9.

20. Post C, Dutz W, Nasarian I. Endemic Pneumocystis Carinii Pneumonia in South Iran. Arch Dis Child 1964:39:35-40.

21. Post C, Fakouhi T, Dutz W, Bandarizadeh B, Kohout EE. Prophylaxis of epidemic infantile pneumocystosis with a 20:1 sulfadoxine+pyrimethamine combination. Curr Ther Res Clin Exp. 1971;13(5):273-9.

22. Masur H, Ognibene FP, Yarchoan R, Shelhamer JH, Baird BF, Travis $\mathrm{W}$, et al. CD4 counts as predictors of opportunistic pneumonias in human immunodeficiency virus (HIV) infection. Ann Intern Med.1989;111(3):223-31.

23. Strategies for prophylaxis of Pneumocystis carinii pneumonia In: Girard PM, Bozzette SA editors. . Pneumocystis carinii. 1995 pp. 551-71.

24. Struijk GH, Gijsen AF, Yong SL, Zwinderman AH, Geerlings SE, Lettinga KD, et al. Risk of Pneumocystis jiroveci pneumonia in patients long after renal transplantation. Nephrol Dial Transplant. 2011;26(10):3391-8.

25. Medrano FJ, Montes-Cano M, Conde M, de la Horra C, Respaldiza $\mathrm{N}$, Gasch A, et al. Pneumocystis jirovecii in general population. Emerg Infect Dis. 2005;11(2):245-50.

26. Maskell NA, Waine DJ, Lindley A, Pepperell JC, Wakefield AE, Miller RF, et al. Asymptomatic carriage of Pneumocystis jiroveci in subjects undergoing bronchoscopy: a prospective study. Thorax. 2003;58(7):594-7.

27. Rabodonirina M, Vanhems P, Couray-Targe S, Gillibert RP, Ganne $\mathrm{C}$, Nizard N, et al. Molecular evidence of interhuman transmission of Pneumocystis pneumonia among renal transplant recipients hospitalized with HIV-infected patients. Emerg Infect Dis. 2004;10(10):1766-73.

28. Fishman JA. Prevention of infection caused by Pneumocystis carinii in transplant recipients. Clin Infect Dis. 2001;33(8):1397-405.

29. Rezavand B, Hosseini MJ, Izadi M, Mahmoodzadeh Poornaki A Sadraei J, Einollahi B, et al. Lethal Pneumocystis jiroveci pneumonia 24 Years After Kidney Transplantation. Nephrourol Mon. 2014;6(2):e13605.

30. Khodadadi H, Mirhendi H, Mohebali M, Kordbacheh P, Zarrinfar $\mathrm{H}$, Makimura K. Pneumocystis jirovecii Colonization in Non-HIV Infected Patients Based on Nested-PCR Detection in Bronchoalveolar Lavage Samples. Iran J Public Health. 2013;42(3):298-305.

31. Innis MA, Gelfand DH, Sninsky JJ, White TJ. PCR protocols: a guide to methods and applications.San Diego: Academic press; 1990.

32. Miller RF, Ambrose HE, Wakefield AE. Pneumocystis carinii f. sp. hominis DNA in immunocompetent health care workers in contact with patients with P. carinii pneumonia.J Clin Microbiol. 2001;39(11):3877-82.

33. Bartlett MS, Vermund SH, Jacobs R, Durant PJ, Shaw MM, Smith JW, et al. Detection of Pneumocystis carinii DNA in air samples: likely environmental risk to susceptible persons. J Clin Microbiol. 1997;35(10):2511-3. 